\title{
Ultrahigh resolution mass spectrometry
}

\author{
Philippe Schmitt-Kopplin • Norbert Hertkorn
}

Published online: 26 September 2007

(C) Springer-Verlag 2007

The integration of soft ionization technologies and ultrahigh resolution FTICR mass spectrometry (Fourier transform ion-cyclotron MS) has enabled the examination of molecules, directly from mixtures, with ultrahigh mass resolution and sub-ppm mass accuracy. This ability to observe structure and reactivity aspects of intact (macro)molecules has, in particular, contributed to the molecular-level characterization of complex matrices, for example novel materials and biological and biogeochemical samples. Here, thousands of molecular formulae can be determined in a single measurement directly from mixtures.

The exceptional performance of ultrahigh-resolution FTICR mass spectroscopy in the characterization of complex systems suffers much less from the detrimental effects of intrinsic averaging, which is a characteristic of most other methods of organic structural spectroscopy. This novel information-rich and direct perception has the potential to entirely change the way we address the molecular-level analysis of complex materials, systems, and processes in general, thereby opening novel perspectives in data-driven systems biology and "omics" approaches.

The inauguration of Europe's first 12 Tesla FTICR mass spectrometer at the GSF National Research Center for Environment and Health provided the opportunity to hear

\footnotetext{
P. Schmitt-Kopplin $(\varangle) \cdot$ N. Hertkorn

GSF Research Center for Environment and Health, Institute of Ecological Chemistry (IOEC), Ingolstädter Landstraße 1, P.O. Box 1129, 85758 Neuherberg, Germany

e-mail: schmitt-kopplin@gsf.de

N. Hertkorn

e-mail: hertkorn@gsf.de
}

expert views on novel research dedicated to the molecularlevel characterization of complex systems. A dozen presentations organized in four sessions were delivered during the "First International Symposium on UltrahighResolution Mass Spectrometry for the Molecular Level Analysis of Complex (BioGeo)Systems", held at the GSF on November 6 and 7, 2006 (www.gsf.de/FTMS2006).

In session one, General Foundations of FTICR Mass Spectrometry, Alan G. Marshall (National High Magnetic Field Laboratory, Tallahassee, USA) provided a wideranging overview about the currently available opportunities of FTMS in molecular-resolution analysis: High-field Fourier transform ion cyclotron resonance mass spectrometry: a platform for "omics". Then, Eugene N. Nikolaev (Institute of Biochemical Physics, Moscow, Russian Federation) investigated the complex trajectories of ions in FTMS cells: The new possibilities in ion clouds dynamic simulation using supercomputers. Application to FTICR and quadrupole devices. Arnd Ingendoh (Bruker Daltonics, Bremen, Germany) provided an overview about current and foreseeable trends in modern mass spectrometry: Mass spectrometry and beyond-new areas of applications.

Session two, From Biological Chemistry to Chemical Biology, focused on life sciences applications. Jeremy K. Nicholson (Division of Surgery, Oncology, Reproductive Biology and Anaesthetics, Imperial College, London, UK) produced a comprehensive assessment about integrated views in "omics" sciences (Metabonomics and top-down systems biology: from personalized healthcare to molecular epidemiology) and Hannelore Daniel (Technical University Munich, Weihenstephan, Germany) complemented this interesting and versatile perspective with The bottom-up approach: from individual genes to the systems biology level of nutrition. Michael Sattler (European Molecular Biology Laboratory, Heidelberg, Germany and GSF Research Center 
for Environment and Health) illustrated the power of modern NMR methods capable of producing well resolved three-dimensional structures of large biomolecules including description of dynamics and reactivity: NMR to study molecular recognition and dynamics of biomacromolecules.

Session three, Novel MS Approaches in Biochemistry and Medicine, illustrated novel MS approaches by Gary Siuzdak (Scripps Center for Mass Spectrometry, La Jolla, USA): Novel mass-based approaches to proteomics and metabolomics and advanced carbohydrate analysis by Jasna Peter-Katalinić (Institute für Medical Physics and Biophysics, Münster, Germany): Requirements on FTICR mass spectrometry for glycomics in structural biology and medicine.

Session four, Repetitive and Non-Repetitive Complex Systems, focused on proteome and complex biogeochemical mixture analysis. Michael Przybylski (Department of Chemistry, University of Konstanz, Germany) elucidated aspects of advanced protein analysis High resolution FTICR mass spectrometry and affinity-proteomics: powerful tool for elucidation of antigen-antibody recognition structures and vaccine development in molecular immunology and complex biogeochemical materials and William T. Cooper (Department of Chemistry and Biochemistry, FSU, Tallahassee, USA) provided insights into the structure, synthesis and degradation of natural organic matter: Insights into organic chemistry of the natural environment; FTICR mass spectrometry of really complex mixtures. Norbert Hertkorn (GSF Institute of Ecological Chemistry, Neuherberg, Germany) described the specific utility and complementarity of NMR and mass spectrometry in the analysis of complex biogeochemical systems: Mapping the molecular compositional space with mass spectrometry, and Philippe Schmitt-Kopplin (GSF Institute of Ecological Chemistry, Neuherberg, Germany) provided a comprehensive assessment of biogeochemical analysis with a variety of current examples: Molecular-level analysis of nonrepetitive structures in biogeosystems.

To recognize the importance of the technique and provide the reader of the journal Analytical and Bioanalytical Chemistry with a perspective of the current trends in FTICR mass spectrometry, this special issue on "Ultrahigh Resolution Mass Spectrometry" was prepared to include research, perspective, and review articles from experts in the field.
This special issue reflects the wide scope of current FTICR mass spectrometry utilization in the analytical characterization of natural products, biomolecules and biogeochemical mixtures, hyphenation of separation and mass spectrometry, and integration of spectroscopy and separation in the molecular-level characterization of complex systems.

At this point, we would like to thank all the authors for their high-quality articles, all referees for the rapid evaluations.

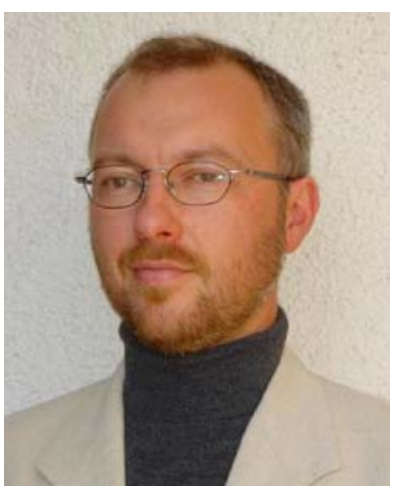

Philippe Schmitt-Kopplin is leader of the molecular BioGeonomics Group at the GSF Institute for Ecological Chemistry in Neuherberg, Germany. He has a long experience in the field of capillary separation sciences with a more recent emphasis on the coupling to mass spectrometry. Other research efforts are directed at the development of novel powerful research tools enabling the targeted and non targeted analysis of complex mixtures such as in metabolomics, and the structural investigation of non-repetitive structures from different environments.

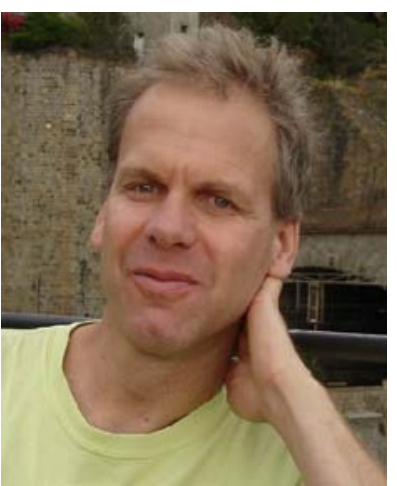

\section{Norbert Herkorn}

is member of the molecular BioGeonomics Group at the GSF Institute for Ecological Chemistry in Neuherberg, Germany. His current research interests are focused on molecular-level structural characterization of natural organic matter and other complex, non-repetitive systems by a combination of NMR spectroscopy, mass spectrometry, separation and combined mathematical data evaluation; the investigation of molecular mechanisms of the interaction of NOM with organic and inorganic pollutants, mineral surfaces and living systems; plant and microbial metabolism; authentification of the origin of natural compounds with isotope-specific methods and biotic and abiotic enantioselectivity. 